\title{
The static dipole polarizabilities of helium and molecular hydrogen by differential diffusion Monte Carlo
}

\author{
C. Huiszoon \\ Department of Physics, University of Twente, P.O. Box 217, 7500 AE Enschede, The Netherlands
}

and

W.J. Briels

Department of Chemistry, University of Twente, P.O. Box 217, 7500 AE Enschede, The Netherlands

Received 31 July 1992; in final form 8 December 1992

\begin{abstract}
The differential diffusion Monte Carlo method, involving correlated random walks, is used to calculate the static polarizabilities of molecular hydrogen and helium by application of a finite electrostatic field. The results are for molecular hydrogen $\alpha_{\perp}=4.60(3)$ au; $\alpha_{\|}=6.38(5)$ au; for helium $\alpha=1.38(1)$ au. The results agree, within the statistical errors, with those obtained by application of high quality ab initio methods.
\end{abstract}

\section{Introduction}

Accurate ground-state energies have been calculated for a number of small molecules and some first row elements employing the diffusion Monte Carlo (DMC) method for solving the many-body Schrödinger equation. The procedure has been described in detail by several authors: Anderson [1], Moskowitz et al. [2], Reynolds et al. [3], and Wells $[4,5]$. A very instructive introduction is also given in Koonin's book [6], which also presents a complete computer program for molecular hydrogen and helium. The development and applications of quantum Monte Carlo have been given in a recent review paper by Lester and Hammond [7].

The energy of a molecule, for a fixed position and orientation, in a homogeneous electric field can be written, as in ref. [8],

$E=E^{(0)}-\mu_{\alpha}^{(0)} F_{\alpha}-\frac{1}{2} \alpha_{\alpha \beta} F_{\alpha} F_{\beta}+\mathcal{O}\left(F^{3}\right)$,

where $E^{(0)}$ is the energy of the free molecule, $\mu_{\alpha}^{(0)}$ is the permanent dipole moment of the molecule in the $\alpha$ direction, $\alpha_{\alpha \beta}$ is the polarizability describing the distortion of the molecule by the external electric field
$\boldsymbol{F}$. Repeated subscripts imply a summation over all Cartesian components. Adding a potential energy term, corresponding with a homogeneous external electrostatic field, $\boldsymbol{F}$, to the Hamiltonian offers the possibility to calculate $E$. For instance the molecular dipole moment of $\mathrm{LiH}$ has been evaluated by Wells [5] from the difference between the molecular energies with and without an external homogeneous electrostatic field. The method described in the present work is an extension of Wells' method towards the calculation of polarizabilities. The DMC method gives energies which are subject to statistical uncertainties associated with the use of random numbers. These variances are at the $10^{-4}$ hartree level, as are the changes in energy, due to molecular polarization in the presence of an electrostatic field of magnitude of, say, 0.005 au, a value that will be used in this work. Therefore these variances hamper the accurate evaluation of the relatively small energy differences. In the calculation of the permanent dipole moment of $\mathrm{LiH}$, Wells introduced two related systems, referred to as the "model" and the "differential" system, both simulated using the same set of random numbers leading to energy differences being free, at 
least to first order, from the statistical variance associated with the energies of the separate systems. Recently a related method, with application to $\mathrm{LiH}$, has been described by Vrbik, Legare and Rothstein [9].

The goal of the present Letter is to show that the so-called differential diffusion Monte Carlo method can also be used for the evaluation of static dipole polarizabilities of molecular hydrogen and helium. Although these two systems have no permanent dipole moments, the configurations, generated during the simulation, will generally correspond with charge distributions having dipole moments different from zero. The energies associated with the presence of thesc instantancous dipole moments in the extcrnal electrostatic field will average out to zero for infinite simulation times, for finite times variances in these energies will surmount the energies associated with the molecular polarizability by several orders of magnitude. This problem has been solved by considering the energy of each configuration in an electric field, $F$ and, at the same time, in the opposite electric field, $-\boldsymbol{F}$. By averaging the two corresponding energies the contributions of the instantaneous dipole moments cancel, at least in first order, and the weighted energy difference can be attributed fully to the polarizability. Mathematically the procedure is equivalent with considering the Cartesian $y z, z x$, and $x y$ planes as mirror planes when the field is along the $x, y$, and $z$ direction, respectively, and by evaluating the energy differences of the energies corresponding to configurations related by a mirror plane. As polarization is a phenomenon due to distortion of the molecular electron cloud in the presence of a field, it is interesting to note that nevertheless the polarizability can be calculated from configurations undistorted by the electric field; the effect of the field enters the calculations through the weight factors used in the energy calculations. Details of the method will be given in section 2 . The dynamic dipole polarizability of the helium atom has been calculated by Caffarel and Hess [10] using the quantum Monte Carlo technique as described by the same authors in ref. [11]. They express the second-order RayleighSchrödinger perturbation contribution to the energy of the system in terms of a two time correlation function of the perturbing operator. The relation be- tween the present approach and that of Caffarel and Hess will be pointed out below.

\section{The diffusion Monte Carlo method}

In diffusion Monte Carlo the Schrödinger equation for the electronic motion is written in imaginary time (atomic units will be used throughout) as follows:

$\frac{\partial}{\partial t} \phi(\boldsymbol{r}, t)=\frac{1}{2} \nabla^{2} \phi(\boldsymbol{r}, t)+\left[E_{\mathrm{T}}-V(\boldsymbol{r})\right] \phi(\boldsymbol{r}, t)$,

where $r$ is a $3 n$-dimensional vector specifying the coordinates of the $n$ electrons of the molecule, $t$ is the imaginary time, $V(\boldsymbol{r})$ is the Coulomb potential energy of the electrons. The "trial energy", $E_{\mathbf{T}}$, is continuously adjusted, during the calculation, to approach the ground-state energy $E^{(0)} . \phi(r, t)$ is the unknown time-dependent wavefunction. The nuclei are positioned at fixed points in space. Eq. (2) is a diffusion equation in a $3 n$-dimensional space, and as such may be simulated by a random-walk process of an ensemble of $N$ configurations, each configuration being a point in $3 n$ space. Efficient sampling is done by means of an importance function $\psi_{\mathrm{T}}(\boldsymbol{r})$, so that the following distribution is generated:

$f(\boldsymbol{r}, t)=\phi(\boldsymbol{r}, t) \psi_{\mathrm{T}}(\boldsymbol{r})$.

This is seen as follows. Multiplying eq. (2) by $\psi_{\mathrm{T}}(\boldsymbol{r})$ and rewriting it in terms of $f(r, t)$ gives an equation for $f(r, t)$,

$$
\begin{aligned}
& \frac{\partial}{\partial t} f(\boldsymbol{r}, t)=\frac{1}{2} \nabla^{2} f(\boldsymbol{r}, t)-\nabla\left(\frac{1}{\psi_{\mathrm{T}}(\boldsymbol{r})} \nabla \psi_{\mathrm{T}}(\boldsymbol{r})\right) f(\boldsymbol{r}, t) \\
& \quad+\left[E_{\mathrm{T}}-E_{\mathrm{L}}(\boldsymbol{r})\right] f(\boldsymbol{r}, t),
\end{aligned}
$$

where $E_{\mathrm{L}}(\boldsymbol{r})$ is the "local energy", $E_{\mathrm{L}}(\boldsymbol{r})=H \psi_{\mathrm{T}}(\boldsymbol{r}) /$ $\psi_{\mathrm{T}}(r)$. The evolution of $f(r, t)$ over a short time $\tau$ from $t$ to $t+\tau$ can be calculated by means of the following approximation for the Green function that holds for small values of the time step $\tau$ :

$$
\begin{aligned}
& G\left(\boldsymbol{r} \rightarrow \boldsymbol{r}^{\prime}, \tau\right)=(2 \pi \tau)^{-3 n / 2} \exp \left\{-\tau\left[E_{\mathrm{L}}\left(\boldsymbol{r}^{\prime}\right)-E_{\mathrm{T}}\right]\right\} \\
& \quad \times \exp \left(\frac{-\left[\boldsymbol{r}-\boldsymbol{r}^{\prime}-\tau \nabla \ln \left|\psi\left(\boldsymbol{r}_{\mathrm{T}}\right)\right|\right]^{2}}{2 \tau}\right),
\end{aligned}
$$

such that $f$ at $\boldsymbol{r}^{\prime}$ and at time $t+\tau$ is obtained from 
$f\left(\boldsymbol{r}^{\prime}, t+\tau\right)=\int \mathrm{d} \boldsymbol{r} G\left(\boldsymbol{r} \rightarrow \boldsymbol{r}^{\prime}, \tau\right) f(\boldsymbol{r}, t)$.

Eq. (6) allows, by repeated application, the evolution of $f(r, t)$ towards its asymptotic form for large values of $t$,

$f(\boldsymbol{r}, t)=\phi_{0}(\boldsymbol{r}) \psi_{\mathrm{T}}(\boldsymbol{r}) \exp \left[-\left(E^{(0)}-E_{\mathrm{T}}\right) t\right]$,

where $\phi_{0}$ is the time-independent ground-state wavefunction. For $E_{\mathrm{T}}=E^{(0)}$ one obtains the steady-state distribution

$f_{\infty}(\boldsymbol{r})=\phi_{0}(\boldsymbol{r}) \psi_{\mathbf{T}}(\boldsymbol{r})$.

The ground-state energy of the system is obtained as the average of the local energy $E_{\mathrm{L}}$ over the distribution $f_{\infty}(\boldsymbol{r})$. The same distribution is also used when the electric field is applied to the system which means that the configurations are not distorted by the electric field. The trial wavefunction used for hydrogen was taken from ref. [6],

$\Psi_{\mathrm{T}}\left(\boldsymbol{r}_{1}, \boldsymbol{r}_{2}\right)=\phi\left(\boldsymbol{r}_{1}\right) \phi\left(\boldsymbol{r}_{2}\right) \chi\left(r_{12}\right)$,

where $\phi$ is a molecular orbital for which a linear combination of two atomic Slater orbitals centered at the protons was chosen,

$\phi\left(r_{i}\right)=\exp \left(-r_{i L} / a\right)+\exp \left(-r_{i R} / a\right)$.

The parameter $a$ is chosen to avoid the electron-nucleus singularity. $\chi$ is the Jastrov factor

$\chi(r)=\exp \left(\frac{r}{\alpha(1+\beta r)}\right)$,

where $\alpha=2$ and $\beta$ is a free parameter that can be optimized by requiring that the variational quantum Monte Carlo energy is a minimum. For hydrogen with an internuclear separation of $1.4 \mathrm{bohr}$ we used $\beta=0.65 \mathrm{bohr}^{-1}$. For helium the function $\phi\left(r_{i}\right)$ consists of only one of the two terms of eq. (10) as for helium $r_{i \mathrm{~L}}$ equals $\boldsymbol{r}_{i \mathrm{R}}$. For helium the value $\beta=0.158$ bohr ${ }^{-1}$ was adopted. The time step, $\tau$, was $0.005 \mathrm{au}$, the field strength $0.005 \mathrm{au}$. The ensemble size was 500.

The presence of a homogeneous electric field, $\boldsymbol{F}$, yields the perturbed Hamiltonian

$H_{ \pm}^{\mathrm{D}}=H^{(0)} \pm F \cdot \boldsymbol{r}$

where $H^{(0)}$ is the Hamiltonian of the free molecule, the \pm sign refers to the two opposite directions of the electric field for which the calculations are done.

The contribution of the ensemble of $N$ configurations to the energy of polarization, $\Delta E=E-E^{(0)}$, after $M$ moves of each configuration, can be written as follows:

$$
\begin{aligned}
\Delta E & =\frac{1}{M} \sum_{k=M 0}^{M+M_{0}-1}\left[\left(\sum _ { i = 1 } ^ { N } w _ { i k } \left\{w_{i k}^{\prime}\left[E_{\mathrm{L}}\left(\boldsymbol{r}_{i k}\right)+E_{\mathrm{F}}\left(\boldsymbol{r}_{i k}\right)\right]\right.\right.\right. \\
& \left.\left.+w_{i k}^{\prime}{ }^{-1}\left[E_{\mathrm{L}}\left(\boldsymbol{r}_{i k}\right)-E_{\mathrm{F}}\left(\boldsymbol{r}_{i k}\right)\right]\right\}\right) \\
& \times\left(\sum_{i=1}^{N} w_{i k}\left(w_{i k}^{\prime}+w_{i k}^{\prime-1}\right)\right)^{-1} \\
& \left.-\sum_{i=1}^{N} w_{i k} E_{\mathrm{L}}\left(\boldsymbol{r}_{i k}\right)\left(\sum_{i=1}^{N} w_{i k}\right)^{-1}\right],
\end{aligned}
$$

where $E_{\mathrm{F}}(\boldsymbol{r})$ is the contribution to the energy of the configuration $r$ due to the presence of the field $\left(E_{\mathrm{F}}(\boldsymbol{r})=\boldsymbol{F} \cdot \boldsymbol{r}\right)$. The weight factors are

$w_{i k}=\exp \left(-\sum_{j=1}^{k} E_{\mathrm{L}}\left(\boldsymbol{r}_{i j}\right) \tau\right)$

and

$w_{i k}^{\prime}=\exp \left(-\sum_{j=1}^{k} E_{\mathrm{F}}\left(\boldsymbol{r}_{i j}\right) \tau\right)$

When only few steps are done, i.e. when the value of $k$ is small, the weight factors $w_{i k}^{\prime}$ and $w_{i k}^{\prime-1}$ in eq. (13) will both be close to unity leading to a zero value for the energy difference $\Delta E$. Therefore it is necessary to do a certain number of thermalization steps before starting the calculation of the energy difference $\Delta E$, in eq. (13) this is indicated by $M_{0}$.

In the actual calculations the weighting was not done as indicated above by using the weight factors $w_{i k}$, but instead a weight factor

$\omega_{i k}=\exp \left[-E_{\mathrm{L}}\left(r_{i k}\right) \tau\right]$

was used in the calculation of the energies and subsequently an integer multiplicity factor $J$ was used for branching

$\left.J=\mathrm{INT}\left\{\exp \left[-E_{\mathrm{L}}\left(\boldsymbol{r}_{i k}\right)+E_{\mathrm{T}}\right)\right] \tau+\xi\right\}$,

where $\xi$ is number taken from a rectangular distri- 
bution of random numbers between 0 and 1 . If $J$ is zero, the configuration is deleted from the ensemble, in the other cases $J-1$ copies of the configuration are added to the ensemble. If the number of configurations in the ensemble differs from the initial size of the ensemble, configurations are randomly deleted or doubled to keep the average size of the ensemble constant.

Once having established the value of $\Delta E$, the components of the polarizability tensor follow from

$\alpha_{\alpha \alpha}=-2 \Delta E / F_{\alpha}^{2}$.

It may be interesting to note that for small values of the exponents in eq. (14b), eq. (13) yields the same expression was used by Caffarel and Hess [10] for the special case of the static dipole polarizability. This can be seen as follows. For small values of the exponents, the following approximations can be made:

$w_{i k}^{\prime}+w_{i k}^{\prime-1}=2$

and

$w_{i k}^{\prime}-w_{i k}^{\prime-1}=-2 \sum_{j=1}^{k} E_{\mathrm{F}}\left(r_{i j}\right) \tau$.

Substitution of eqs. (18a) and (18b) into eq. (13) yields

$$
\begin{aligned}
& \Delta E=-\tau \frac{1}{M} \\
& \times \sum_{k=M_{0}}^{M+M_{0}-1}\left[\left(\sum_{i=1}^{N} w_{i k} \sum_{j=1}^{k} E_{\mathrm{F}}\left(\boldsymbol{r}_{i j}\right) E_{\mathrm{F}}\left(\boldsymbol{r}_{i k}\right)\right)\left(\sum_{i=1}^{N} w_{i k}\right)^{-1}\right] \\
& \\
& \approx-\tau\left\langle\sum_{i=1}^{K} E_{\mathrm{F}}\left(\boldsymbol{r}_{j}\right) E_{\mathrm{F}}\left(\boldsymbol{r}_{K}\right)\right\rangle_{\psi \mathrm{T}},
\end{aligned}
$$

where in the right-hand part of eq. (19), the set of $N$ configurations has been replaced by one single configuration, and the corresponding index, $i$, has been dropped. The angular brackets denote the average over a large set of subsequent trajectories of the configuration with $T=K \tau, K \approx M+M_{0}-1$, as their simulation time and with $\psi_{\mathrm{T}}$ as the guiding function for the random walks. The right-hand side of eq. (19) can be written as the integral

$\Delta E=-\int_{0}^{T}\left\langle E_{\mathrm{F}}(\boldsymbol{r}(t)) E_{\mathrm{F}}(\boldsymbol{r}(T))\right\rangle_{\psi \mathrm{T}} \mathrm{d} t$.
As the two time correlation function in eq. (20) only depends on the difference between the arguments $t$ and $T$, one can write

$$
\begin{aligned}
\Delta E & =-\int_{0}^{T}\left\langle E_{\mathrm{F}}(\boldsymbol{r}(0)) E_{\mathrm{F}}(\boldsymbol{r}(T-t))\right\rangle_{\psi \mathrm{T}} \mathrm{d} t \\
& =-\int_{0}^{T}\left\langle E_{\mathrm{F}}(\boldsymbol{r}(0)) E_{\mathrm{F}}(\boldsymbol{r}(u))\right\rangle_{\psi \mathrm{T}} \mathrm{d} u,
\end{aligned}
$$

which leads for large values of $T$, using eq. (17), to expression (12) for the static $(\omega=0)$ dipole polarizability in ref. [10].

Fig. 1 shows the typical behavior of the accumulative energy difference, eq. (13), in a plot against the number of moves per configuration in an ensemble of average size of 500 configurations. The field strength was $0.005 \mathrm{au}$, the time step $0.005 \mathrm{au}$. From fig. 1 it is seen that the accumulative energy difference becomes stable after, say, 2000 moves, the value thus chosen for $M_{0}$. The actual calculations were done as follows. After doing a number of thermalization moves, typically 10000 moves per configuration, a loop was started to evaluate the energy differences. First 1000 moves per configuration of the ensemble were done, without the presence of the electric field to avoid serial correlation between the subsequent evaluations of the energy difference $\Delta E$. Then, according to the above discussion, $M_{0}$ moves, in the presence of the field, were done to reach the regime

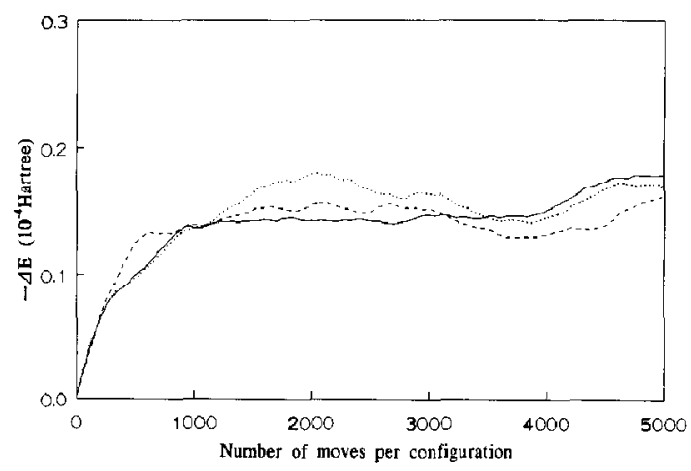

Fig. 1. The accumulative energy difference as a function of the number of moves per configuration in an ensemble of average size 500 configurations. Field strength 0.005 au, time step 0.005 au for the helium atom. The three curves refer to the three Cartesian directions. 
where the energy differences are stable. Subsequently 400 moves per configuration of the ensemble were done to obtain an estimate of the energy difference $\Delta E$. This calculation of the estimate for $\Delta E$ is repeated 600 times to reduce and estimate the statistical variance of the average, $\overline{\Delta E}$.

\section{Results of the calculations}

Fig. 2 shows the value for the quantity $-2 \overline{\Delta E} / F^{2}$, calculated for helium and plotted as a function of $M_{0}$. The three curves correspond with the electric field, $\boldsymbol{F}$, directed along the three Cartesian directions. It is seen that the quantity $-2 \overline{\Delta E} / F^{2}$ reaches a stationary value after a sufficient number of thermalization moves have been done. This stationary value of $-2 \overline{\Delta E} / F^{2}$ is the polarizability $\alpha$, eq. (1). Fig. 3 gives similar results for molecular hydrogen. Here the $x$ and $y$ directions are perpendicular to the molecular axis, the $z$ direction is parallel to the molecular axis. The origin is at the molecular center. The present calculated polarizabilities have been listed in table 1 together with results obtained by other authors. It may be seen that the values we have obtained fully agree with those obtained by other ab initio methods that can be expected to give exact results. It is also seen that the statistical error

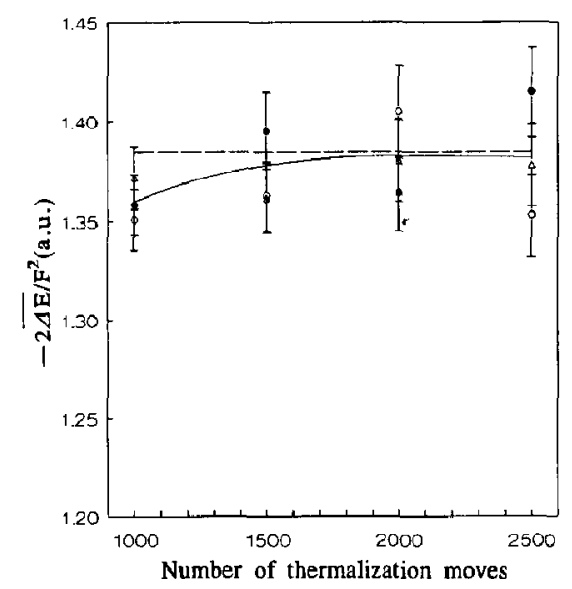

Fig. 2. Helium: $-2 \overline{\Delta E} / F^{2}$ as a function of the second number of thermalization moves. The three curves correspond with an external electric field directed along the three Cartesian directions, (C) $x,(0) y$ and $(\Delta) z$. The dashed line corresponds with the polarizability taken from ref. [15] and quoted in table 2 .

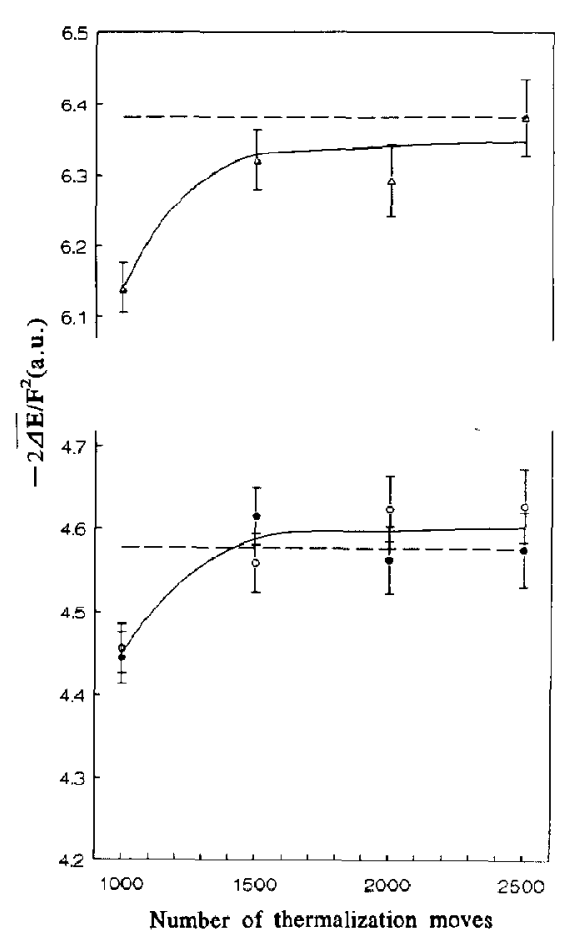

Fig. 3. Hydrogen: $-2 \overline{\Delta E} / F^{2}$ as a function of the second number of thermalization moves. The three curves correspond with an external electric field directed along the three Cartesian directions. The $x(\bullet)$ and $y(0)$ directions are perpendicular to the molecular uxis, the $z(\Delta)$ direction is parallel to the axis. The dashed lines correspond to the values of the polarizabilities taken from ref. [19] and quoted in table 1.

we found in our calculations is relatively large. Probably this error can be reduced by increasing, for instance, the ensemble size and the number of evaluations of the energy difference $\Delta E$. This will require more CPU time. For a typical run, about $40 \mathrm{~h} \mathrm{CPU}$ time on the Convex C240 machine of the University of Twente computational center, are required.

The choice of the field strength is not critical. No dependence of the quantity $-2 \overline{\Delta E} / F^{2}$, and its variance, has been detected for a field strength 0.0025 au. The independence indicates that the energy difference is entirely due to the dipole polarizability.

\section{Conclusions}

The results presented here show that the diffusion Monte Carlo solution to the many-body Schrödinger 
Table 1

Polarizabilities of helium and molecular hydrogen in au

\begin{tabular}{|c|c|c|c|}
\hline Helium & & & $\alpha$ \\
\hline Rayleigh-Schrödinger perturbation theory & & & $1.380^{\mathrm{a})}$ \\
\hline explicitly correlatcd result & & & $1.383^{\mathrm{b})}$ \\
\hline time-dependent coupled Hartree-Fock & & & $1.322^{\mathrm{c})}$ \\
\hline full configuration interaction & & & $1.385^{\mathrm{d})}$ \\
\hline single double triple many body perturbation theory & & & $1.354^{\circ)}$ \\
\hline rigorous upper and lower bounds & & & $1.38355(76)^{f)}$ \\
\hline quantum Monte Carlo & & & $\left.1.38(2)^{8}\right)$ \\
\hline present work & & & $1,38(1)$ \\
\hline experiment & & & $1.397(1)^{\text {h) }}$ \\
\hline Molecular hydrogen ${ }^{\text {i) }}$ & $\alpha_{\perp}$ & $\alpha_{\|}$ & $\bar{\alpha}^{j)}$ \\
\hline variation perturbation method & 4.57769 & 6.38049 & $5.17862^{k)}$ \\
\hline Rayleigh-Schrödinger perturbation theory & 4.570 & 6.441 & $5.194^{\mathrm{a})}$ \\
\hline present work & $4.60(3)$ & $6.38(5)$ & $5.19(3)$ \\
\hline
\end{tabular}

a) Ref. [12]. b) Ref. [13]. ") Ref. [14]. 'Ref. [15]. "Ref. [16]. ') Ref. [17]. ") Ref. [10].

h) Calculated from the value of the Clausius-Mossotti function listed in ref. [18].

i) Internuclear separation 1.4 bohr.

j) $\bar{\alpha}=\left(2 \alpha_{1}+\alpha_{\perp}\right) / 3$.

k) Ref. [19].

equation, using the finite field technique and correlated sampling, can be used to calculate the static dipole polarizabilities of helium and molecular hydrogen which agree with the best ab initio results.

\section{Acknowledgement}

Thanks are due to Dr. M. Caffarel for very useful remarks.

\section{References}

[1] J.B. Anderson, J. Chem. Phys. 63 (1975) 1499.

[2] J.W. Moskowitz, K.E. Schmidt, M.A. Lee and M.H. Kalos, J. Chem. Phys. 77 (1982) 349.

[3] P.J. Reynolds, D.M. Ceperley, B.J. Alder and W.A. Lester Jr., J. Chem. Phys. 77 (1982) 5593.

[4] B.H. Wells, in: Methods in computational chemistry, Vol. 1, ed. S. Wilson (Plenum Press, New York, 1987).

[5] B.H. Wells, Chem. Phys. Letters 115 (1985) 89.
[6] S.E. Koonin, Computational physics (Addison-Wesley, New York, 1986) ch. 8.

[7] W.A. Lester Jr. and B.L. Hammond, Ann. Rev. Phys. Chem. 41 (1990) 283.

[8] A.D. Buckingham, Advan. Chem. Phys. 12 (1967) 107.

[9] J. Vrbik, D.A. Legare and S.M. Rothstein, J. Chem. Phys. 92 (1991) 1221

[10] M. Caffarel and O. Hess, Advances in Biomolecular Simulations, Obernal, France, AIP Conf. Proc. No. 239 (1991) 20.

[11] M. Caffarel and O. Hess, Phys. Rev. A 43 (1991) 2139.

[12] W. Meyer, Chem. Phys. 17 (1976) 27.

[13] A.J. Thakkar, J. Chem. Phys. 75 (1981) 4496.

[14] F. Visser, P.E.S. Wormer and P. Stam, J. Chem. Phys. 79 (1983) 4973.

[15] F. Visser, P.E.S. Wormer and W.P.J.H. Jacobs, J. Chem. Phys. 82 (1985) 3753.

[16] W. Rijks and P.E.S. Wormer, J. Chem. Phys. 88 (1988) 5704.

[17] R.M. Glover and F. Weinhold, J. Chem. Phys. 65 (1976) 4913.

[18] O.R. Johnston, G.J. Oudemans and R.H. Cole, J. Chem. Phys. 33 (1960) 1310.

[19] W. Kolos and L. Wolniewicz, J. Chem. Phys. 46 (1967) 1426. 